\title{
Cambio químico a través de recetas de cocina
}

\section{Chemical change through cooking recipes}

DOI: $10.46932 / \mathrm{sfjdv} 2 \mathrm{n} 2-078$

Received in: March 1st, 2021

Accepted in: May 30th, 2021

\author{
Laura Andrea Ruiz Sepúlveda \\ Secretaria de educación de Bogotá. \\ E-mail: laruizs1@educacionbogota.edu.co
}

\begin{abstract}
RESUMEN
La presente propuesta didáctica corresponde a la síntesis de un trabajo realizado entre 2013 y 2014 con estudiantes de grado $10^{\circ}$ y $11^{\circ}$ en Bogotá y presentado en el encuentro pedagógico de Cuba 2017. Por medio de él se aborda el concepto de cambio químico en educación media, reconociendo los fundamentos que hacen de este eje central en la enseñanza de la química, en la fase inicial se reconocen las ideas previas identificando los errores conceptuales más comunes, después de aplicar las diferentes actividades didácticas se identifican las modificaciones en los errores conceptuales de los estudiantes. Las 5 actividades didácticas realizadas se presentan en forma alternativa a las prácticas de laboratorio tradicionales, manejando herramientas de la vida cotidiana, así como recetas de cocina que son de fácil asociación y aplicación para los estudiantes; superando las dificultades de acceso a laboratorios y los riesgos que presentan algunas aulas de química que no están adecuadas para trabajar con sustancias químicas (manejo de reactivos, campanas de extracción, etc.) de igual manera en la actualidad este tipo de actividades pueden brindar una alternativa para el trabajo remoto realizado en casa. La propuesta permite ver la química de una manera más asequible relacionándola con la solución de problemas sencillos, superando la visión tradicional y cerrada de la ciencia.
\end{abstract}

Palabras Claves: Cambio químico, Aprendizaje activo, Caja didáctica, Ideas previas, errores conceptuales, química y cocina.

\begin{abstract}
This didactic proposal corresponds to the synthesis of a work carried out between 2013 and 2014 with 10th and 11th grade students in Bogota and presented at the pedagogical meeting in Cuba 2017. Through it, the concept of chemical change in secondary education is approached, recognizing the fundamentals that make this central axis in the teaching of chemistry, in the initial phase the previous ideas are recognized identifying the most common conceptual errors, after applying the different didactic activities the modifications in the conceptual errors of the students are identified. The 5 didactic activities carried out are presented as an alternative to traditional laboratory practices, handling tools of daily life, as well as cooking recipes that are easy for students to associate and apply; overcoming the difficulties of access to laboratories and the risks presented by some chemistry classrooms that are not adequate for working with chemical substances (handling of reagents, extraction hoods, etc.) in the same way, nowadays this type of activities can provide an alternative for remote work done at home. The proposal allows to see chemistry in a more accessible way, relating it to the solution of simple problems, overcoming the traditional and closed vision of science.
\end{abstract}

Keywords: Chemical change, active learning, didactic box, previous ideas, conceptual errors, chemistry and cooking. 


\section{METODOLOGÍA}

Se tiene en cuenta como modelo pedagógico el constructivismo,el modelo didáctico a trabajar es aprendizaje activo, el estudiante es un sujeto activo que posee unos esquemas, conceptos o representaciones, las cuales están condicionadas por la percepción que esteposee de la realidad, cuando su interpretación del mundo cambia también lo hacen sus constructos mentales, el trabajo se desarrolló en las siguientes etapas:

- Indagación: Se realizó una revisión bibliográfica del concepto cambio químico.

- Identificación de ideas previas: A partir de la indagación se recopilaron las ideas previas que se manejan en torno al concepto "cambio químico" para identificar dichas ideas en los grupos objeto de estudio, se elaboró un cuestionario de ideas previas por medio del aplicativo en la página de la asociación Americana para el avance de la ciencia (AAAS) ${ }^{1}$ el cual se empleó antes para reconocer los errores conceptuales que manejaban los estudiantes y después de la intervención en el aula para identificar lasideas que habían sufrido transformaciones.

- Caracterización de los grupos: los grupos se seleccionaron teniendo en cuenta que el eje temático "cambio químico" se suele trabajar en los grados decimó y once.

- Selección de experiencias: Con base en la bibliografía consultada y las ideas previas identificadas con el cuestionario inicial se analizaron varias actividades, cada una buscaba abordar uno o varios saberesfundamentales en el aprendizaje de cambio químico.

- Diseño caja didáctica: Teniendo en cuenta la bibliografía consultada, se seleccionaron 5 actividades prácticas que se adaptaron a la metodología deaprendizaje activo. Se considera como caja didáctica al conjunto de materiales y guías que orientan cada experiencia.

\section{ESTRATEGIAS Y ACTIVIDADES}

Estrategias sociales: el aprendizaje colaborativo facilita la inclusión de los estudiantes al sistema educativo, esta estrategia se ha aplicado en el aula, ya que la institución recibe constantemente estudiantes desplazados y de otras minorías poblacionales, que al entrar en un grupo de trabajo fortalecen sus lazos de amistad, desarrollan habilidades comunicativas y superan más fácilmente los problemas académicos y convivenciales.

Actividades pedagógicas: En el aprendizaje activo las experiencias se clasifican endos grandes

\footnotetext{
${ }^{1}$ En el marco del proyecto financiado por NSF 2061, la Asociación Americana para el Avance de la Ciencia (AAAS) tiene una colección de 600 artículos clasificados por recursos y temas los elementos están diseñadospara estudiantes de secundaria y preparatoria y están planteados en torno a conceptos erróneos estudiantiles documentados, los productos se pueden consultar en la página http://assessment.aaas.org/pages/home, con las herramientas de esta página se elaboró un cuestionario que posteriormente se tradujo y aplico a los estudiantes objeto de estudio.
} 
grupos, demostraciones interactivas cuando el docente realiza la demostración, actividades experimentales cuando son los estudiantes quienes realizan las prácticas; esta clasificación también se tuvo en cuenta en el momento de organizar las actividades. En el siguiente cuadro se muestra un esquema sobre la clasificación de las actividades y los aspectos que se tuvieron en cuenta. 
1Tabla:: Actividades Pedagógicas.

\begin{tabular}{|c|c|c|c|}
\hline Actividad & Saber & $\begin{array}{l}\text { Tipo de } \\
\text { experiencia. }\end{array}$ & $\begin{array}{l}\text { Ideas previas } \\
\text { Obstáculos epistemológicos } \\
\text { (a superar). }\end{array}$ \\
\hline Alcohol y fuego & $\begin{array}{c}\text { Cambio físico vs cambio } \\
\text { químico. } \\
\text { Combustión. } \\
\text { Cambio de estado. }\end{array}$ & Demostrativa & $\begin{array}{l}\text { Desaparición de la materia. } \\
\text { Cambio de estado igual a } \\
\text { cambio químico. } \\
\text { Continuidad. } \\
\text { Sustancialismo. }\end{array}$ \\
\hline $\begin{array}{l}\text { Mayonesa y } \\
\text { gelatina. }\end{array}$ & $\begin{array}{l}\text { Mezclas } \\
\text { Emulsiones y coloides. } \\
\text { Fuerzas } \\
\text { intermoleculares }\end{array}$ & Experimental & Cambio químico como mezcla. \\
\hline $\begin{array}{c}\text { Manzanas } \\
\text { Acarameladas }\end{array}$ & $\begin{array}{l}\text { Combustión } \\
\text { oxidación } \\
\text { Caramelización. } \\
\text { Carbonización. }\end{array}$ & Experimental & $\begin{array}{l}\text { Flogisto. } \\
\text { El oxígeno no participa. } \\
\text { Transmutación de la naia }\end{array}$ \\
\hline Arepuelas & $\begin{array}{lr}\text { Reacciones } & \text { con } \\
\text { desprendimiento degas, } & \text { de } \\
\text { Reacciones } & \mathrm{y} \\
\text { neutralización } & \mathrm{de} \\
\text { Reacciones } & \\
\text { descomposición. } & \end{array}$ & Demostrativa & $\begin{array}{c}\text { Productos preexistentes. } \\
\text { Flogisto. }\end{array}$ \\
\hline $\begin{array}{l}\text { Bebida de Limón y } \\
\text { bicarbonato. }\end{array}$ & $\begin{array}{l}\text { Reacciones con } \\
\text { desprendimiento de } \\
\text { gas. } \\
\text { Ecuación química. } \\
\text { Ley de las proporciones } \\
\text { definidas. }\end{array}$ & Experimental & $\begin{array}{l}\text { Productos preexistentes. } \\
\text { Flogisto. } \\
\text { Sustancias que desaparecen. }\end{array}$ \\
\hline
\end{tabular}

\section{EVALUACIÓN Y SEGUIMIENTO}

En el desarrollo de las experiencias se tuvo en cuenta la bibliografía consultada (Bonwell) (Intitut de Ciéncies de 1’Educacio. Universitat Politecnica de Cataluya) Las actividades prácticas seleccionadas se adaptaron a la metodología de aprendizaje activo, orientadas en ocho momentos.

\section{Planteamiento del problema}

2. Predicciones individuales: Se pide a los estudiantes pensar sobre los posibles resultados de la experiencia y registrar su predicción individual.

3. Predicciones de grupo: Los estudiantes discuten sus predicciones en un pequeño grupo de 3 o 4 llegando de ser posible a un consenso.

4. Registro de predicciones grupales: Cada grupo nombra un relator que registra la predicción final del grupo.

5. Socialización de predicciones: El docente recoge (verbalmente o por escrito) las predicciones de cada grupo sin hacer juicio de valor sobre la validez delas predicciones. Las predicciones de grupo, no son las del relator y no tienen calificación.

6. Evidenciar o realizar la experiencia: El docente realiza la demostraciónmostrando claramente los 
resultados, o los estudiantes realizan la práctica registrando los resultados.

7. Descripción y discusión de resultados: Se pide a algunos estudiantes que para toda la clase, describan los resultados y los discutan en el contexto de la actividad. Se confrontan las predicciones con las observaciones.

8. Síntesis y extrapolación de resultados: Los estudiantes (o el docente) realizan una síntesis de los conceptos involucrados en los resultados anteriormente analizados. Además, se puede utilizar para discutir situaciones análogas con diferentes características superficiales (diferentes situaciones, que responden al mismo concepto(s)).

Al finalizar la aplicación de las experiencias se empleó de nuevo el cuestionariode ideas previas al grupo para comparar el estado inicial de las ideas de los estudiantes con el estado final de las mismas y determinar si las actividades habían modificado los errores conceptuales.

\section{ANALISIS DE RESULTADOS.}

1 Imagen :practica Manzanas caramelizadas.

2 Imagen :practica Arepuelas.

3 Imagen :Gelatina y mayonesa.
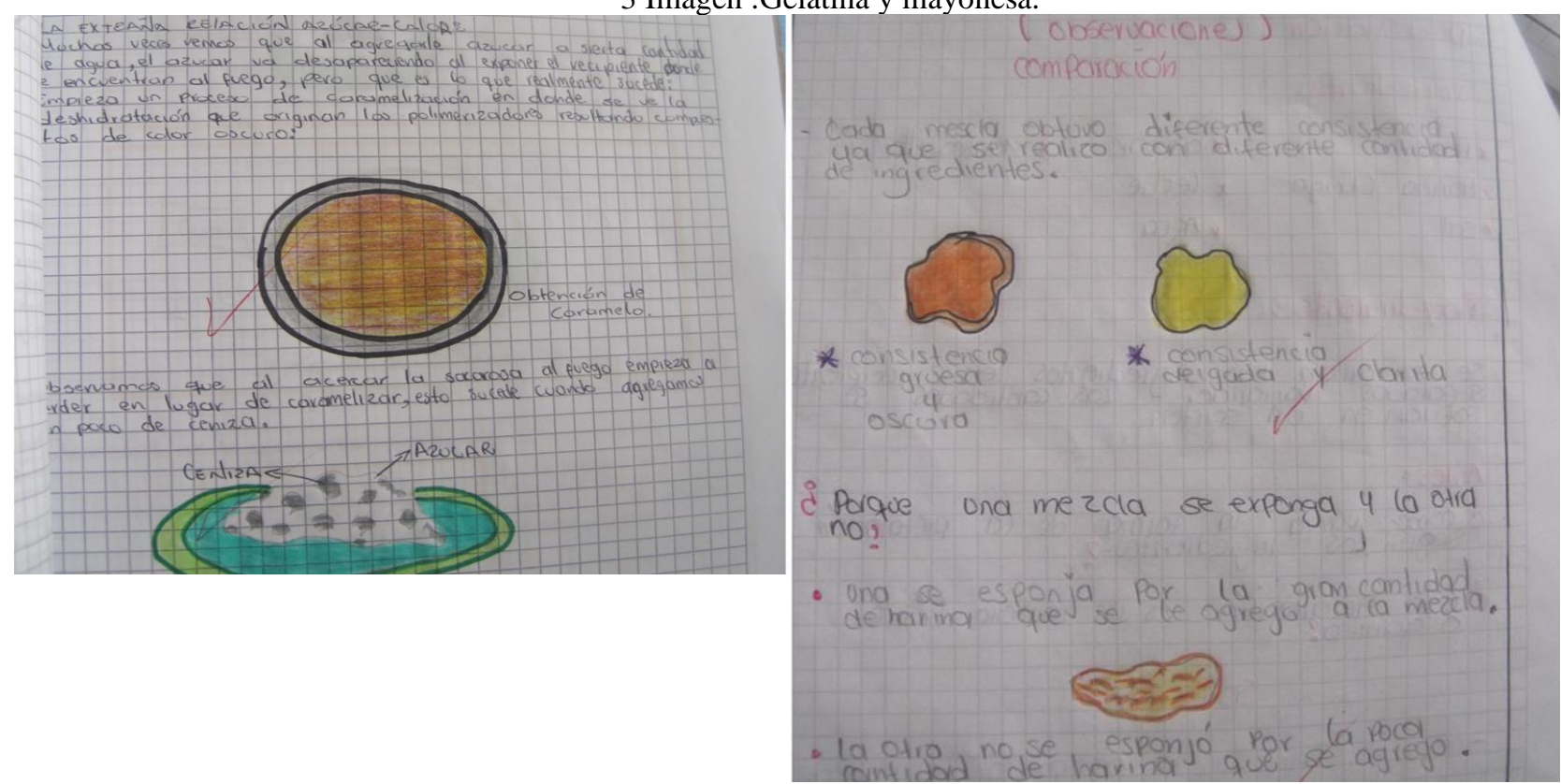


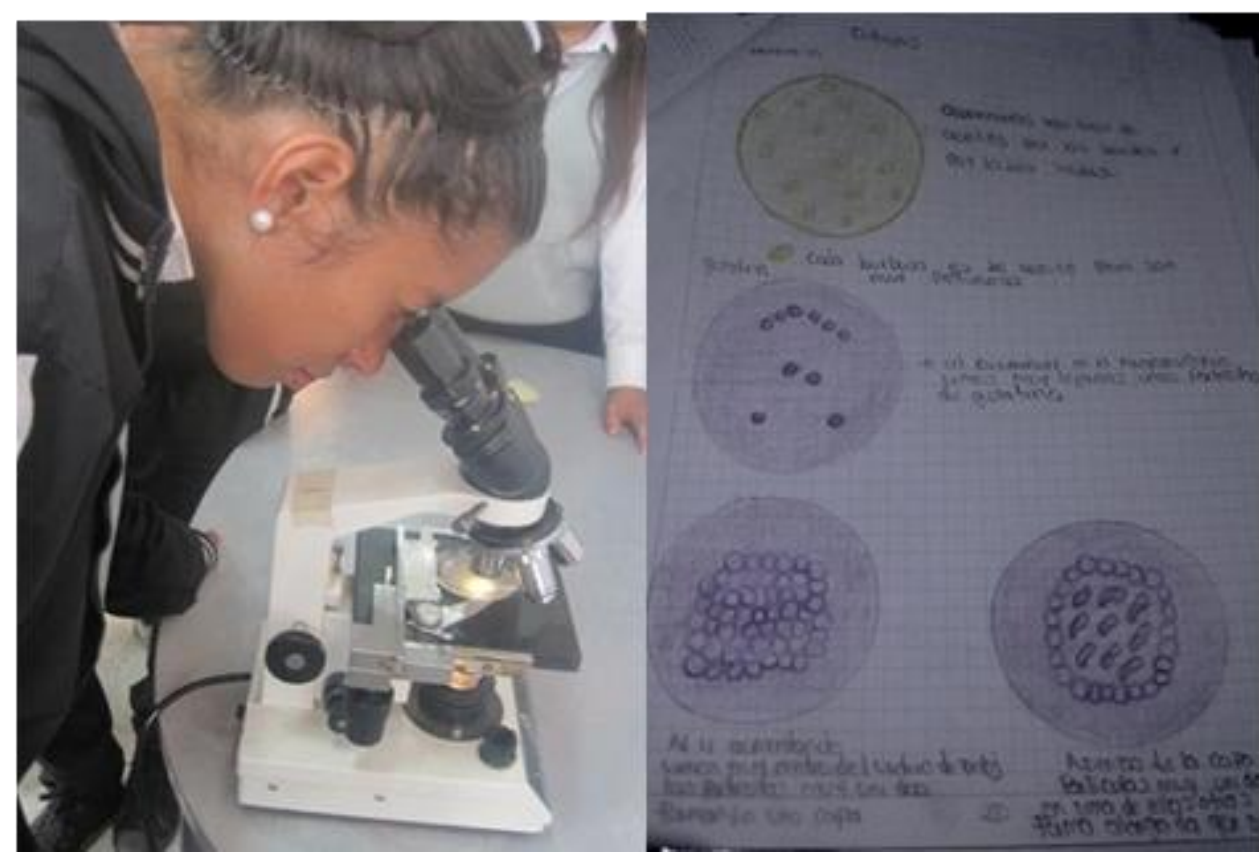

En la práctica de manzanas caramelizadas los estudiantes reconocieron fácilmente cambios físicos como disolver o cortar, pero les costó un poco más de análisis determinar si el cambio de color o forma representaban un cabio físico o químico, otros estudiantes relacionaron los cambios irreversibles con el cambio químico (por ejemplo, cuando el caramelo por exceso de energía sufre el proceso de carbonización.

Se puede observar la persistencia de errores conceptuales comunes; en la practica de alcohol y fuego se evidencia la existencia de ideas similares al flogisto, (materia que desaparece o gana y pierde peso) para muchos el fuego es un cambio de estado del alcohol, otro error conceptual común es identificar solamente los cambios visibles (macro) y desconocer los procesos no visibles (micro), en la práctica de las arepuelas muy pocos estudiantes identificaron el papel del oxígeno en la forma final de la masa, la mayoría consideraron que para que la masa se esponjara, influía la cantidad de harina u otros ingredientes.

La practica de gelatina y mayonesa buscaba enfrentar a los estudiantes con la idea de materia corpuscular, muchos de ellos esperaban observar en el microscopio moléculas y átomos, en su lugar vieron como las partículas de una mezcla y un coloide se organizaban, para ellos fue difícil encontrar las palabras para describir lo observado a través del microscopio utilizaron palabras como pequeñas gotas, puntos , partes etc.

Por otra parte la práctica de bicarbonato y limón puso en evidencia la interacción directa de dos sustancias y muchos pudieron concluir que correspondía a un cambio químico, sin embargo la representación de la ecuación constituía una dificultad para los estudiantes. 
Las actividades practicas vinculadas con recetas de cocina, permiten al estudiante relacionar los conceptos de la vida cotidiana con la ciencia, promueve la motivación y la indagación. El aprendizaje activo crea espacios para el análisis y la contrastación de hipótesis.

\section{CONCLUSIONES}

- $\quad$ Al aplicar el cuestionario de ideas previas (diseñado con el instrumento de la AAAS) se determinó que en el grupo objeto de estudio los errores conceptuales más recurrentes relacionados con cambio químico, se podían agrupar en tres categorías: Conservación de la materia, cambio físico vs cambio químico y propiedades de la materia. Al aplicar la prueba $\mathrm{t}$ a los resultados generales del cuestionario inicial y final se pudo observar un nivel de significancia del 0,025 , el cual nos permite rechazar la hipótesis nula y considerar que los cambios en el cuestionario fueron resultado de la aplicación de las actividades didácticas en el aula.

- $\quad$ El aprendizaje activo como estrategia didáctica permite conocer los errores conceptuales (a través de las predicciones) intervenir en ellos y reconocer las modificaciones que los estudiantes alcanzan, el trabajo colaborativo como apoyo del aprendizaje activo, le da a los estudiantes espacios para discutir, construir y reconstruir significados, además hace posible una evaluación constructiva pues son los mismos estudiantes quienes reconocen sus fortalezas y debilidades en el proceso.

- Al comparar las ideas de los estudiantes antes y después de aplicar las actividades prácticas, se pudieron encontrar las siguientes reflexiones: Se logró que un porcentaje significativo de estudiantes superara la confusión entre cambio de estado y cambio químico. Mejoro el reconocimiento de cambios químicos. Se identifica correctamente la conservación de los átomos.

\section{ANEXO 1}

\section{GUIA MANZANAS ACARAMELADAS}

Tipo de actividad: practica experimental

Dirigida a: estudiantes grado decimo (educación media, ciclo v)

Tiempo estimado: 1 hora y 30 minutos

\section{Introducción}

Los cambios químicos se encuentran presentes en nuestra cotidianidad, pero si bien sabemos que existen en ocasiones es difícil reconocer cuando un fenómeno observado corresponde a un cambio 
químico. Por medio de la siguiente práctica los estudiantes podrán reconocer los cambios físicos y químicos que ocurren en lapreparación de un postre, identificando fenómenos como, oxidación, combustión, caramelización y carbonización; como parte central de la receta está la obtención de caramelo lo cual permite analizar las propiedades de la sacarosa.

\section{Objetivos}

- Identificar cambios físicos y químicos en la preparación.

- Explicar los procesos de oxidación, combustión, caramelización y carbonización.

\section{Materiales}

- 6 manzanas

- 1 taza de azúcar

- 1 taza de agua

- 3 astillas de canela

- El jugo de 4 limones.

- Recipiente metálico

- Cuchara metálica

- Capsulas de porcelana

- Pinzas para capsula

- Estufa (presente en el laboratorio)

\section{Planteamiento del problema}

Se prepararán manzanas caramelizadas teniendo en cuenta los siguientes pasos

1. Pelar las manzanas.

2. sumergir 4 de ellas en una taza de agua con jugo de 6 limones,

3. las otras 2 dejarlas expuestas al aire,

4. Preparar en una olla el caramelo calentando $1 / 2$ taza de azúcar disuelta conuna taza de agua, y astillas de canela, revolver de manera constante por 10 minutos hasta que espese.

5. Cortar 4 rodajas de manzana y colocarlos en un plato.

6. Con una cuchara de metal tomar 4 muestras de caramelo y calentarlas directamente a la llama agregando el resultado a las tajadas de manzanapuestas en los platos

\section{A) 20 segundos}


6 B) 40 segundos

6 C) 1 minuto

6 D) Hasta carbonizaciónDejar

enfriar.

7. Tomar dos capsulas de porcelana

7A agregar en una un poco de azúcar, calentarlo con el mechero utilizandopinzas. (No dejar quemar)

7B en la otra capsula agregar un poco de caramelo resultante de la práctica, calentarlo con el mechero utilizando pinzas. (No dejar quemar)

\section{Predicciones individuales y grupales}

Se pedirá a los estudiantes que realicen las siguientes predicciones en formaindividual.

1. ¿Qué sucederá con las manzanas que se sumergen en el jugo de limón ylas que no se sumergen en el jugo de limón?

2. ¿Cómo será la consistencia del caramelo?

3. ¿Cambiara la consistencia del caramelo cuando se enfrié? ¿Por qué?

4. ¿El azúcar y el Caramelo son la misma sustancia? Si/ No ¿Por qué? Luego se reunirán en grupo para establecer consensos sobre las predicciones ynombrar un relator.

Socialización de predicciones

Los grupos socializaran sus predicciones y estas se registraran en el tablero delaula de clase. Realización de la receta

Cada grupo realizara la receta, utilizando los materiales de la caja didáctica ysiguiendo el procedimiento indicado allí.

Descripción y discusión

1. En la siguiente tabla indique para cada paso si corresponden a cambiosquímicos o físicos y explique por qué.

\begin{tabular}{|c|c|c|c|}
\hline & $\begin{array}{c}\text { Cambio } \\
\text { Físico }\end{array}$ & $\begin{array}{c}\text { Cambio } \\
\text { químico }\end{array}$ & Explicación \\
\hline 1 & & & \\
\hline 2 & & & \\
\hline
\end{tabular}


2. ¿De qué depende que la consistencia del caramelo sea dura o blanda?

3. ¿Al calentar el azúcar y el caramelo con el mechero? ¿Se puede concluirque son las mismas sustancias?

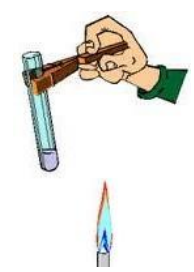

\section{Síntesis y extrapolación de resultados.}

Con base en la discusión y los análisis hechos por los estudiantes, el maestro identificara los cambios físicos y químicos ocurridos durante la práctica. Luego se explicara las principales características del limón y la sacarosa destacando que lasfrutas sufren un proceso de oxidación cuando se exponen al aire y que el limón actúa como antioxidante, en el azúcar al agregar energía se forma una nueva sustancia conocida como caramelo, al agregar mayor energía se puede llegar a la carbonización completa con la producción de dióxido de carbono y agua, pormedio de las ecuaciones el maestro puede mostrar que ocurre es una reacomodación de los átomos formando nuevas sustancias.

En la explicación realizada por el maestro se pueden incluir otros procesos de oxidación y combustión, igualmente se pueden introducir otros procesos químicos como la fermentación y la descomposición, finalmente los estudiantes pueden realizar un cuadro comparativo entre cambio físico y cambio químico.

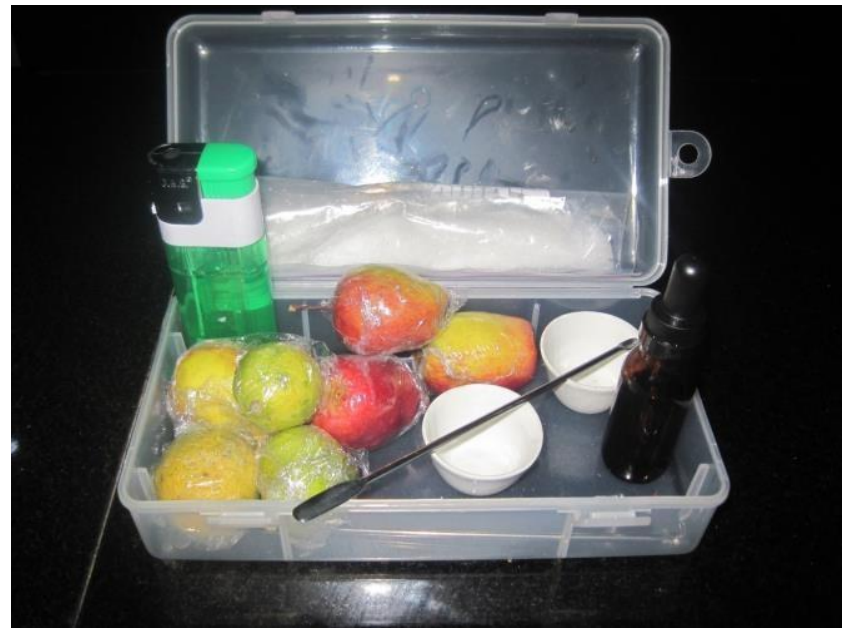




\section{BIBLIOGRAFIA}

AAAS. (s.f.). Sience Assessmente. http://assessment.aaas.org/pages/home.

Barcena, A. \&. (2007). Real sociedad Española de química. Recuperado el 8 de Marzo de 2013, de El azúcar en la enseñanza secundaria: http://www.rseq.org

Intitut de Ciéncies de 1’Educacio. Universitat Politecnica de Cataluya. (s.f.). El aprendizaje Activo. Una nueva forma de enseñar y aprender.

Lacolla, L. (Junio de 2012). La representación social que los estudiantes poseen acerca de las reacciones químicas y su incidencia en la construcción del concepto de cambio químico. Tesis Doctoral. Burgos.

Martín del Pozo, R. (Julio de 1998). La construcción Didactica del concepto cambio químico. Alambique(17), 65-75.

Prieto, T. \&. (2007). Trabajo Practico y concepciones de los alumnos: La combustion. En M. A. Izquierdo (Ed.), Investigar en la enseñanza de la química. Nuevos horizontes: contextualizar y modelizar (Primera ed., págs. 115-141). Barcelona, España: Universidad autonoma de Barcelona.

Raviolo, A. A. (2011). Sustancia y reacción química como conceptos centrales en química. Eureka, $8(3), 240-254$.

Ruiz, L ( 2013) . Aprendizaje activo de cambio químico en educación media por medio de una caja didáctica.

Solsona, N. (Enero de 2010). Una experiencia Competencial de química y bizcochos en el aula. Aula de Innovación educativa(188), 52-55. 